\title{
A Fashion Intelligent Guiding Device Based on Multi-sensor
}

\author{
Li-xia Zhang \\ Department of Electronic and Information Technology \\ Zhengde Polytechnic College \\ Nanjing, 211106, China \\ zhanglixia2000@sina.com.cn
}

\author{
Jia-an Zhang, Xinyi Meng, Ning Liu, Haifeng Liu \\ School of Control Science and Engineering \\ Hebei University of Technology \\ Tianjin, 300130, China \\ zhangjiaan@foxmail.com
}

\begin{abstract}
An intelligent guidance device based on ultrasonic sensor array is researched and developed according to the blind demand for facilitate walking. Six pairs of ultrasonic sensors are divided into the upper and the lower groups to detect obstacles. Multi-source information fusion and multisensor information collected are processed. Through the use of LM35 temperature sensor, measuring accuracy is improved. With fusion processing to detect multi-sensor data, wireless speech segment tips based on Bluetooth technology are achieved. Experimental results show that the effective sensing distance of the device is about $30 \sim 4000 \mathrm{~mm}$ and the relative error of the actual value is within $2.5 \%$, which meets the demands of daily use for the blind.
\end{abstract}

Keywords-multi-sensor; ATmega128A microprocessor; guidance; Bluetooth

\section{INTRODUCTION}

China is emerging about 450000 blind people every year. Predict by 2020 , number of the Chinese vision disability will attain the scale of fifty million ${ }^{[1,2]}$. Increasing the quality of blind man's life and facilitating their travel have been paid social attention by more and more people. In recent years, scholars who researched on the system of humanity and rehabilitation aids gradually increased. Reference [3] developed a kind of ultrasonic guidance system for blind based on the principle of continuous emission frequency modulation, and it provided a theoretical basis and guidance for the further research of guidance system. Through a broadband frequency hopping spread spectrum ultrasonic signal to increase robustness to noise and reverberation, Reference [4] proposed a new ultrasonic ranging method which testing accuracy was better than $0.5 \mathrm{~mm}$ in a typical office environment. The guidance device applied to blind or partially sighted groups needs to effectively guide the blind during the avoidance of obstacles, meanwhile, the device needs to avoid the ordinary consumers are eager but hard to reach just for its expensive price.

The method of using man-machinery interface or piecewise audio prompts to convey a travel information or to avoid obstacle warning information for the blind has corrupt practice ${ }^{[5,6]}$. In order to improve the early warning accuracy, and with the purpose of optimizing the warning information transmission this paper designs a fashion intelligent guiding device based on multi-sensor. The device adopts 6 pairs of ultrasonic sensor which is divided into two groups of compounds to detect the obstacles. With the help of one temperature compensation scheme ${ }^{[7]}$, the ultrasonic signal in the design is temperature compensated with LM35 temperature sensor in order to reduce the measuring blind area. Data fusion technology is used for weighted processing to detect the range of ultrasonic signal obstacle distance in different directions ${ }^{[8]}$. With the help of Bluetooth wireless communication technology ${ }^{[9]}$, wireless speech segment tips on the safe or dangerous obstacle distance are achieved. In order to facilitate the test during the system debugging, TFT LCD screen is used to display detection distance and ambient temperature, which is the auxiliary design. The device has the advantages of high measurement accuracy, high-level humanized service and strong practicability, and hence this device makes a good technology promotion and market application prospect.

\section{System Working PrinciPle ANd Design Scheme}

\section{A. System working principle}

Ultrasonic pulse signal can produce Doppler effect in the case of encountering moving objects, and produce significant reflection that reflects echo signal in the case of encountering impurities or interface. This intelligent guiding device is designed based on echo transit time $[10,11]$. The detected targets of the guidance device is mostly obstacles plane or surface, and the echo signal is mainly from the echo signal which is reflected by the main lobe beam. It is known that the propagation velocity of ultrasonic waves is $344 \mathrm{~m}$ per second in the air. The propagation velocity is expressed in $\mathrm{v}$. If ultrasonic pulse emitted by the sensors to the receiving time is $t$, the s distance from the sensor to the obstacle can be expressed as

$$
s=344 \mathrm{~m} / \mathrm{s} \times t \div 2
$$

As AVR MCU uses the crystal of $11.0592 \mathrm{MHz}$, the time used by MCU on each count once takes approximately $1 \mu \mathrm{s}$. That is to say $t$ equals the number of count. Acoustic wave propagation speed will be affected by the temperature, and the relationship adopts

$$
v=331.4+0.607 \mathrm{~T}
$$

In the equation the unit of $\mathrm{v}$ is $\mathrm{m}$ per second, and $\mathrm{T}$ with the unit of degrees centigrade says the environmental temperature. To reduce the measuring blind area and 
improve the measurement accuracy this system uses the temperature sensor LM35 to structure temperature compensation circuit, for its output voltage is linear relation with the Celsius scale.

\section{B. Design scheme}

Following traditional blind crutch length adjustable and convenient advantages, the total length of the intelligent guiding device designed is about $100 \mathrm{~cm}$. The device is divided into 3 sections, and different sections are fixed by the card hole and button in the tensile state. The first section with hollow interior is connected with a handle, and it can shrink to the second section inward. The second section can also shrink to the third section inward. 3 pairs of ultrasonic sensors that are remembered as the first group are mounted on the second section's external bias above. Combined with characteristics of ultrasonic sensor, the installation angle is about 10 degrees. The first group is to deal with certain height obstacle from the front, front left and right directions. Other 3 pairs of ultrasonic sensors that are remembered as the second group are mounted on the third section's external bias lower. The second group is to deal with relatively low obstacle from the front, front left and right directions. Temperature compensation module, voice module, Bluetooth module and LCD module are fitted on the first section inside and outside. When the sensor obstacle distance is about 200 $\mathrm{cm}$, external voice will prompt the blind to slow down with a slow speed. In the range of $100 \mathrm{~cm}$, the external voice will offer the respective obstacle orientation and prompt the blind to bypass road if the sensors from the first group first sensed obstacles, otherwise, when the sensors from the second group sensed obstacles alone, the external voice will prompt the blind to slow down with rapid speech, and offer the obstacle orientation. In some relatively noisy place, you can switch for Bluetooth earphone voice broadcast mode just by pressing the button on the first section's handle.

Such as the system structure block diagram shown in figure 1 , the guidance device, based on the microprocessor ATmega128A microprocessor as the main control unit, mainly includes the ultrasonic module, voice module, temperature sensor module, TFT module, Bluetooth module, USB/ISP interface, et al.

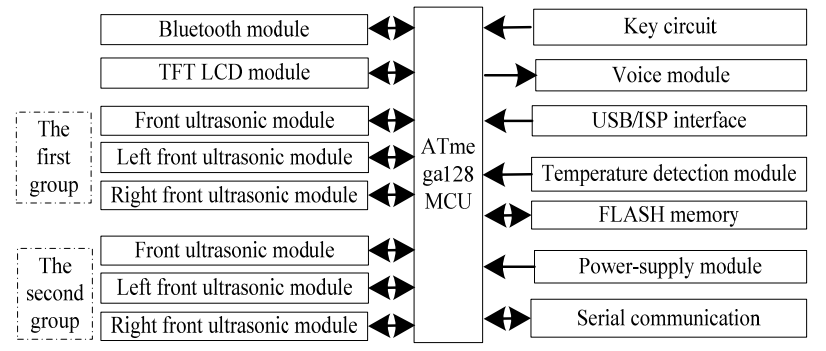

Figure 1. Bloch diagram of the system structure

\section{System Main Module of Hardware Design}

\section{A. Ultrasonic module}

As ultrasonic signals have large attenuation in the air, the echo signal from long distance obstacle will be weak ${ }^{[12]}$. With the reasonable design of echo detection circuit, good ultrasonic transducer determines the effective detection range of ultrasonic detection device and its measurement accuracy ${ }^{[13]}$. This design uses 6 pairs of electrostatic transducer which are divided into two groups to connect with ATmega128A microprocessor. The first group connects with ATmega128A microprocessor from PA0 to PA4, from PC0 to PC4, and from PF0 to PF4. All in all, they use 30 ports. Figure 2 shows the front ultrasonic module in the first group of transmitting and receiving circuit principle diagram. Designing the residual vibration time $2 \mathrm{~ms}$, ATmega128A microprocessor generates ultrasonic signal $40 \mathrm{kHz}$. This device configures the ATmega128A microprocessor from PA0 to PA2 ports to control transmitter unit, and configures PA3 and PA4 ports to control receiver unit.
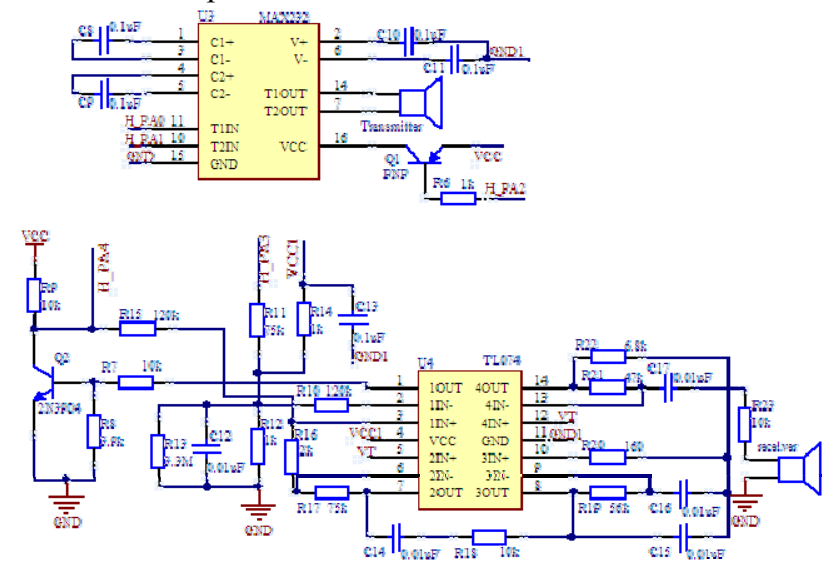

Figure 2. Ultrasonic module circuit in front of first groups

The maximum detectable range of the ultrasonic module is about $5 \mathrm{~m}$, and the precision can reach $0.3 \mathrm{~cm}$, and the blind area is about $2 \mathrm{~cm}$, and the emission diffusion angle is less than 15 DEG. All this is conducive to improve the accuracy of obstacle distance guidance device detection.

\section{B. Voice module}

The voice module uses ATmega128A microprocessor to collect data from the ultrasonic and the input key, and turns this data into judgment signal which enables the voice chip to play the corresponding speech. Figure 3 shows the voice module circuit diagram. This guidance device adopts ISD4004 series single chip voice recording circuit, and configures the ATmega128A chip PB0 port to control ISD4004 chip $\overline{S S}$ port. It also configures the chip PB2 and PB3 ports which respectively connect to ISD in and out ports MOSI and MISO as SPIO port, and configures PD1 port as interrupt port to connect to ISD ${ }^{\overline{I N}} \bar{T}$ port. In figure 3 , the port label H_PD0 is connected to the serial clock SCLK. The serial clock is generated by the master controller and is always consistent with MOSI and MISO ports data 
transmission. Data on the rising edge of SCLK latches on to ISD, and on the trailing edge of SCLK removes off of ISD. The port label H_PD7 which can be used for storage management technology is connected to the ISD row address clock.

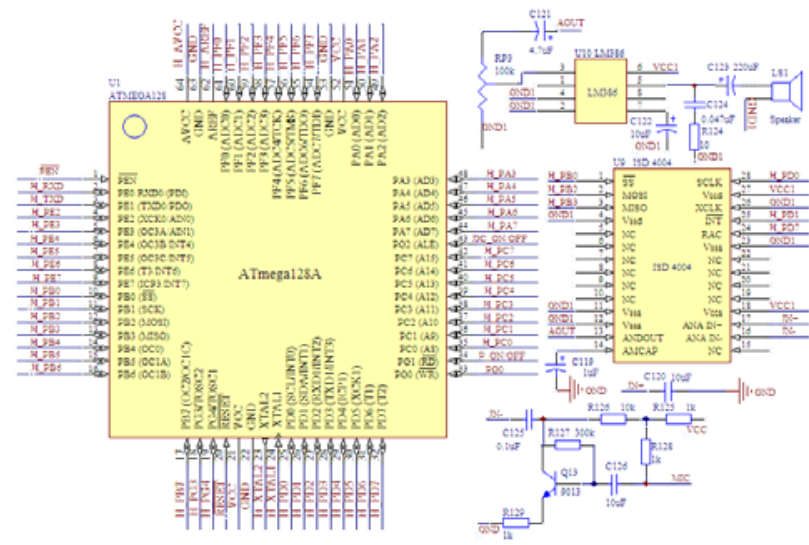

Figure 3. Voice module circuit

\section{Bluetooth module}

With the help of Bluetooth short distance wireless communication technology, Bluetooth headset can realize wireless voice communication between the earphone and audio gateway. The Bluetooth module mainly comprises radio frequency unit, link control unit, and link management unit and software protocol. The guidance device uses CSR's BC215A as the core chip Bluetooth headset. This chip integrates RF transmitter and receiver, frequency synthesizer and IQ regulator. Because the chip function is perfect, only peripheral circuit can be relatively simple to achieve the requirements of radio frequency.

\section{System SOFTWARE DESIGN}

Software development uses the integrated development environment of ATMEL AVR Studio, and uses C language programming which has the advantages of good portability and high efficiency of programming. The system main program flow chart is shown in figure 4 . The $i$ and $j$ among them say ultrasonic sensor number whose values is from 0 to 5 , and is a total of 6 . The main program flow includes system initialization, emitting and receiving ultrasonic signal, judging ultrasonic sensor group and position, and piecewise audio prompt module. The software program design also involves TFT LCD module to display distance, and involves Bluetooth module to open and shut.

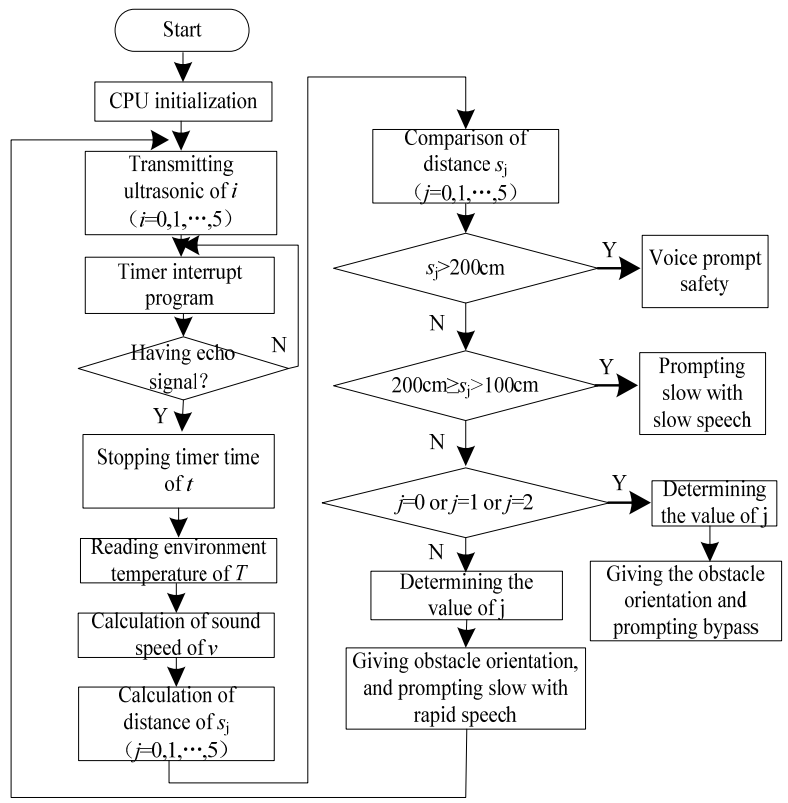

Figure 4. System main program flow chart

\section{System Overall Test}

To test the dependability and practicability of system, the test environment is chosen an indoor environment where is decorated a certain obstacles with the environment temperature of 20 degrees. Compare the obstacle distance measured by tape with the distance on LCD screen configured on the guidance device, and the test results are shown in figure 5 .

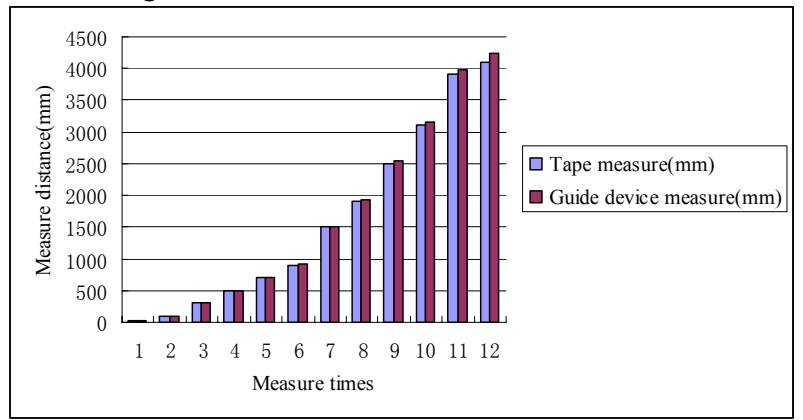

Figure 5. Comparison of test results of obstacle distance

The data can be seen from figure 5 that the actual value of the relative error is within $1 \%$ when the obstacle distance range is from 100 to $900 \mathrm{~mm}$. After repeated testing, the results show that the device can effectively detect obstacles within the range from 30 to $4000 \mathrm{~mm}$. The actual value of the relative error is within $2.5 \%$. The functions of piecewise audio prompt and voice broadcast by Bluetooth headset can be achieved. All of these show that the intelligent guidance device design is successful. 


\section{CONCLUSION}

The intelligent guiding device designed is respectively assembled 3 pairs of ultrasonic sensors in the middle and bottom. The measurement data after data fusion processing determines the piecewise audio prompt hints to the content that can prompt to the blind by Bluetooth headset. Multisensor design can effectively deal with the complex environment of the traffic and road conditions. Optimized installation position can improve the sensing accuracy to the barriers or ladders in the city. Piecewise audio prompt promotes the system that human nature turns more. Wireless voice exchange with the Bluetooth technology can be better used in a noisy place. With the temperature compensation, the system can meet certain measurement accuracy, and can effectively guidance the blind to avoid obstacles. All to these make more independent and freedom to the blind.

\section{REFERENCES}

[1] http://news.xinhuanet.com/society/2006-10/15/content_5204668.htm

[2] Chen Jian-hua, Pan Yu-hong, "Strong or weak: the paradox of Chinese blind social status," Gansu Social Sciences. No.2, pp. 55-58, 2012. (in Chinese)

[3] Wang Yang, Xu Yong, Yang Ju, "Research on the detecting performance of CTFM ultrasonic guidance system for the blind," Electro Acoustic Technology. Vol.36, no.5, pp.66-68,73,2012. (in Chinese)

[4] Saad M M, Bleakley Chris J, Dobson Simon, "Robust high-accuracy ultrasonic range measurement system," IEEE Transactions on
Instrumentation and Measurement. Vol.60, no.10, pp.3334-3341, 2011.

[5] Lee Choon-young, Choi Ho-Gun, Park Jun-Sik, "Collision avoidance by the fusion of different beam-width ultrasonic sensors," 6th IEEE Conference on Sensors. pp.985-988, Oct. 2007.

[6] Calder David J, "Ecological solutions for the blind," 4th IEEE International Conference on Digital Ecosystems and Technologies. pp.625-630, Apr. 2010.

[7] Chen Yi-kang, Zhang Zheng-jie, Chen Ming-rui, "The design of millimeter-level ultrasonic distance measurement circuit," Advanced Materials Research. pp.1260-1265,2012. (in Chinese)

[8] Luo Rui, Zhang Zi-hong, "Study on data fusion algorithm based on ultrasonic sensor of T-R separation," Science Technology and Engineering. Vol.13, no.6, pp. 1585-1587,1591,2013. (in Chinese)

[9] Fan Jing, Yang Rong, "Bluetooth wireless network and A/D converter for data acquisition," Instrument Technique and Sensor. no.z1, pp.261-263,270, 2009. (in Chinese)

[10] Hou He-ping, Liu KAI, Zhang Hai-yan, "Research on new double sheet detection of press and system development," Chinese Journal of SENSORS AND ACTUATORS. Vol.25, no4, pp.552-556, 2012. (in Chinese)

[11] Sun hao, Yi Wei-dong, "A low-power drive circuit design for ultrasonic transducer," Journal of Computer Research and Development. vol.48, no.z2, pp.1-6, 2011. (in Chinese)

[12] Wang Shu-hai, Liu Qiu-zhen, Chen Shu-wang, "Design and application of distance measure ultrasonic sensor," Lecture Notes in Electrical Engineering. vol.178,no.3,pp.109-114, 2013. (in Chinese)

[13] Kang Yi, Sun Shi-chao, "Transmitting /receiving circuit design for ultrasonic based on sing-around velocimeter," Instrument Technique and Sensor. no.1, pp.65-67, 2012. (in Chinese) 